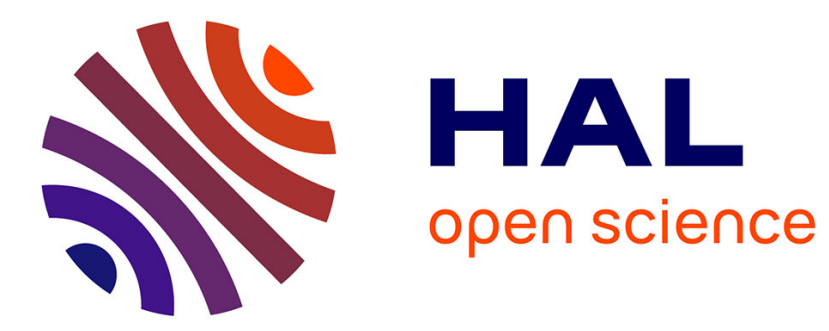

\title{
Les applications de la sensibilité sociale en contexte éducatif 1
}

Pier-Olivier Caron, Jacques Forget, Ariane Leroux-Boudreault

\section{To cite this version:}

Pier-Olivier Caron, Jacques Forget, Ariane Leroux-Boudreault. Les applications de la sensibilité sociale en contexte éducatif 1. Journal de Thérapie Comportementale et Cognitive, 2014, 24 (4), pp.144-150. doi : 10.1016/j.jtcc.2014.07.004. 10.1016/j.jtcc.2014.07.004 . hal-01085090

\section{HAL Id: hal-01085090 https://hal.science/hal-01085090}

Submitted on 20 Nov 2014

HAL is a multi-disciplinary open access archive for the deposit and dissemination of scientific research documents, whether they are published or not. The documents may come from teaching and research institutions in France or abroad, or from public or private research centers.
L'archive ouverte pluridisciplinaire HAL, est destinée au dépôt et à la diffusion de documents scientifiques de niveau recherche, publiés ou non, émanant des établissements d'enseignement et de recherche français ou étrangers, des laboratoires publics ou privés. 


\title{
Les applications de la sensibilité sociale en contexte éducatif ${ }^{1}$
}

\author{
Pier-Olivier Caron ${ }^{2}$, Jacques Forget et Ariane Leroux-Boudreault \\ Département de psychologie, Université du Québec à Montréal
}

Dans un contexte où l'intégration des élèves handicapés ou en difficulté d'adaptation ou d'apprentissage est privilégiée, les enseignants doivent composer avec les défis qu'entraîne cette orientation pédagogique [3-4]. Ces derniers doivent gérer les comportements et les apprentissages de plus d'une vingtaine d'élèves sans pouvoir leur attribuer une attention méticuleuse et individualisée [2]. Une panoplie d'interventions comportementales et cognitives ont été développées afin de répondre à différents problèmes rencontrés par les enseignants (voir, par exemple, [5-6]). Ces interventions validées sur le plan empirique permettent une meilleure gestion de classe. Toutefois, elles exigent souvent une plus grande implication des professionnels du milieu et elles ont parfois le désavantage d'être centrées sur les comportements problématiques de quelques élèves. La question demeure ; comment améliorer les comportements d'un élève sans nuire à la gestion globale de la salle de classe ? Depuis les premiers travaux de Forget [1] sur les applications éducatives de la loi de l'appariement, les enseignants disposent d'un modèle empirique leur permettant d'améliorer leur gestion des comportements difficiles d'un élève sans qu'ils soient dans l'obligation d'attribuer davantage d'attention à ce dernier.

La sensibilité sociale permet d'étudier la relation entre les comportements d'un individu et l'attention sociale prodiguée par son entourage [2]. Le concept repose sur la

\footnotetext{
${ }^{1}$ Référence complète : Caron, P.-O., Forget, J., \& Leroux-Boudreault, A. (2014). Les applications de la sensibilité sociale en contexte éducatif. Journal de Thérapie Comportementale et Cognitive, 24, 144-150. doi: $10.1016 /$ j.jtcc.2014.07.004

${ }^{2}$ Courriel : pocaron19@gmail.com
} 
fonction de l'attention sociale comme stimulus de renforcement [7] et sur la description quantitative de la loi généralisée de l'appariement [8]. Il réfère essentiellement aux choix comportementaux que peut faire une personne par rapport aux contingences de renforcement social disponibles dans son environnement social. En d'autres termes, le modèle prédit, dans un contexte éducatif par exemple, que les comportements d'un élève s'ajusteront en fonction de la distribution relative de l'attention sociale obtenue de son enseignant.

La sensibilité sociale suppose que l'attention est renforçante pour le participant et que les comportements de ce dernier peuvent être décrits adéquatement par la loi de l'appariement. Ces deux hypothèses impliquent que l'attention sociale augmente ou maintienne le taux d'apparition du comportement, peu importe qu'elle puisse sembler négative (réprimandes, isolements ou retrait d'un objet) ou agréable (félicitations, contacts physiques ou obtention d'un privilège) et que la distribution des comportements soit proportionnelle à la quantité d'attention relative qu'il obtient. Ces considérations sont justifiées par l'importante littérature empirique qui appuie l'une et l'autre de ces conceptions (voir [7,9] et plus particulièrement [10] pour une synthèse sur le phénomène). Enfin, si l'une des deux affirmations se révélait fausse, les résultats montreraient que l'inexactitude du modèle et d'autres investigations seraient alors nécessaires. Ces considérations seront développées ultérieurement.

Afin d'illustrer le concept, Forget et Rivard [2] donnent l'exemple d'un enseignant, appelons-le l'enseignant A, attribuant quotidiennement une moyenne de 50 renforçateurs de nature sociale (p. ex. sourire, explication, félicitations) contingents soit à des comportements appropriés, soit à des comportements inappropriés d'un élève. La 
sensibilité sociale propose que l'enfant ajuste la nature et la fréquence de ces comportements en fonction des gains relatifs qu'il peut obtenir. Selon le modèle, si l'enseignant A renforce 25 fois des comportements inappropriés et 25 fois des comportements appropriés, il devrait observer autant de comportements inappropriés qu'appropriés. L'avantage de la sensibilité sociale est de prédire qu'un deuxième enseignant, ici nommé enseignant $\mathrm{B}$, qui renforce en moyenne 25 fois les comportements d'un élève peut être plus efficace que l'enseignant A qui distribue 50 renforçateurs. En effet, si l'enseignant B intervient en moyenne 20 fois auprès des comportements appropriés d'un élève et 5 fois pour les comportements inappropriés (25 stimuli de renforcement au total), il devrait observer que l'élève émet quatre fois plus de comportements appropriés qu'inappropriés. Ainsi, l'enseignant B obtient une meilleure fréquence des comportements adéquats, même s'il attribue moins de renforçateurs aux comportements appropriés.

La sensibilité sociale devient intéressante à évaluer dans un contexte de salle de classe lorsque l'enseignant cherche à modifier les comportements d'un ou de quelques élèves. En effet, dans une telle situation, il est possible de montrer qu'une diminution absolue de son attention peut engendrer une augmentation des comportements appropriés de l'élève si la proportion relative d'attention prodiguée à ces derniers est supérieure à la proportion d'attention accordée aux comportements inappropriés. Autrement dit, la loi généralisée de l'appariement, loi à partir de laquelle est construite la notion de sensibilité sociale ici, affirme que la fréquence d'un comportement est dépendante non pas tant du taux absolu de stimuli de renforcement qui lui est associé, mais bien du taux relatif $[2,8]$. Mathématiquement, la relation d'appariement est représentée par l'équation ; 


$$
\log \left(B_{1} / B_{2}\right)=a \log \left(R_{1} / R_{2}\right)+\log c
$$

où les $B$ s et les $R$ s représentent respectivement la fréquence des comportements et des renforçateurs obtenus par un organisme, où le paramètre $a$ représente sa sensibilité aux contingences de renforcement et où le paramètre $c$ représente sa tendance à émettre tel ou tel comportement. Cette version est appelée la loi généralisée de l'appariement et stipule que le ratio des comportements est proportionnellement relié au ratio des renforçateurs moyennant deux paramètres libres [8-9]. Elle repose sur l'estimation de trois paramètres décrits ci-dessous.

Le premier est la sensibilité, c'est-à-dire la façon dont l'individu ajuste ses choix aux renforçateurs qu'il obtient. Cette sensibilité désigne les changements comportementaux de l'élève en fonction des variations de l'attention sociale prodiguée par l'enseignant. Il s'agit du concept clé au sein de la sensibilité sociale telle qu'abordée ici. Son avantage est de permettre la quantification de l'ajustement comportemental d'un individu par rapport aux contingences de son environnement social. Lorsque cette relation est strictement proportionnelle (sensibilité $=1$ ), la sensibilité est dite théoriquement idéale. La sensibilité peut aussi être inférieure à $1(<1)$, ce qui signifie qu'il faut une plus grande variation de renforçateurs afin d'obtenir une modification comportementale substantielle de l'individu. La sensibilité peut aussi être supérieure à 1 $(>1)$, ce qui signifie qu'une petite variation des contingences de renforcement entraîne une modification importante de la distribution relative des comportements de l'élève.

Le deuxième paramètre est le biais ou la préférence de l'individu à émettre plus fréquemment l'un ou 1'autre comportement en l'absence de renforçateurs. Il s'agit, par 
conséquent, de la tendance «naturelle » de la personne à émettre un certain comportement plutôt qu'un autre. La valeur peut être positive en faveur des comportements appropriés, par exemple, ou négative en faveur des autres comportements (ici, les notions de valeurs négatives et positives sont des définitions arbitraires et ne portent pas de jugement sur la nature du comportement).

Le troisième paramètre est la quantité de variances expliquées, c'est-à-dire à quel point le modèle décrit adéquatement l'ajustement comportemental de l'individu en regard des variations dans le taux d'attention. La sensibilité et le biais sont évalués sans contraintes par rapport aux données empiriques. Par conséquent, il faut mesurer le pourcentage de variance expliquée témoignant du degré auquel le comportement de la personne correspond au modèle de la sensibilité sociale. Cela permet de vérifier la vraisemblance à laquelle les paramètres peuvent être interprétés. La figure 1 illustre un exemple hypothétique afin de présenter graphiquement la sensibilité sociale et chacun des paramètres sous-jacents.

En quoi la sensibilité sociale peut-elle contribuer aux interventions en contexte éducatif ? D’abord, elle permet de conceptualiser la relation élève-enseignant en fonction des relations entre différents comportements et différentes sources de renforcement (p. ex., félicitations, regard, proximité, explication). Il s'agit d'une conception plus contextualiste des contingences de renforcement en salle de classe puisque l'intervention n'insiste pas que sur la relation fonctionnelle entre un seul comportement et un seul renforçateur. Cela permet une interprétation plus précise et adéquate des interactions entre les comportements de l'élève et son enseignant. Ensuite, l'utilisation de la sensibilité sociale valorise des interventions basées sur l'augmentation des 


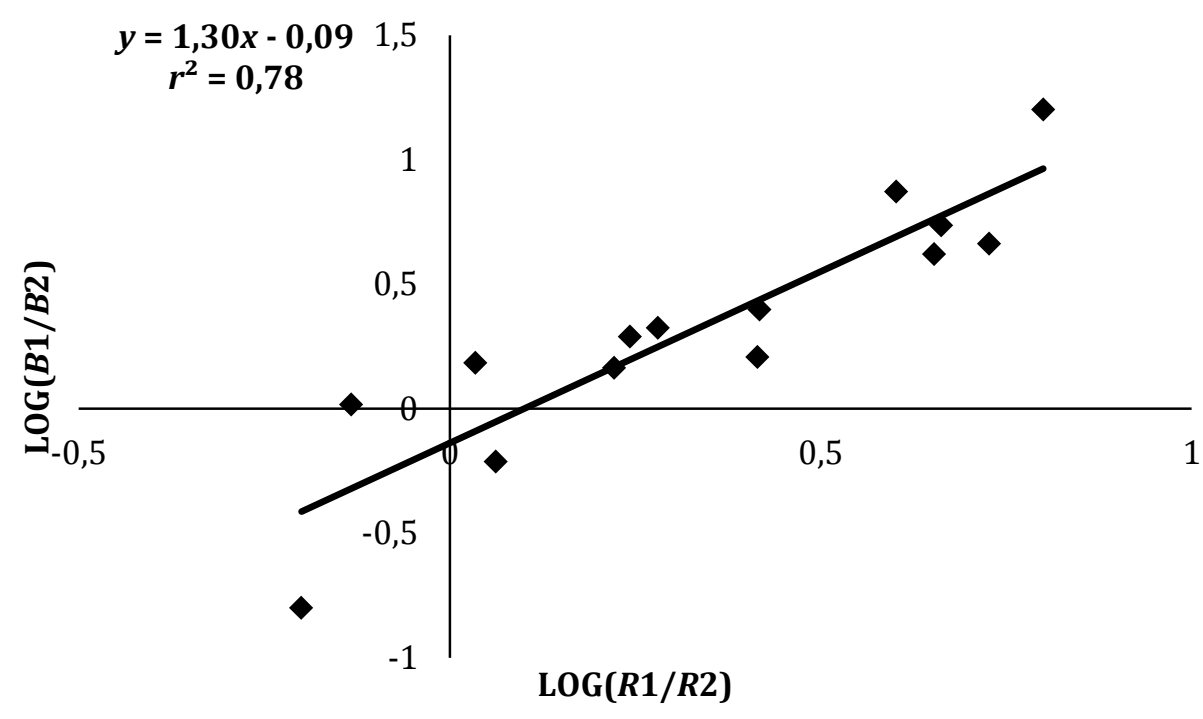

Figure 1. Illustration hypothétique de la sensibilité sociale d'un participant. $B 1$ et $B 2$ symbolisent respectivement les comportements appropriés et inappropriés et $R 1$ et $R 2$ correspond aux renforçateurs sociaux obtenus pour chacun des comportements. La sensibilité est de 1,13 et est représentée par la pente de la droite, le biais est de -0,09 et est représenté par l'ordonnée à l'origine et la variance expliquée $\left(r^{2}\right)$ est de $78 \%$. Le participant est très sensible aux renforçateurs sociaux (sensibilité > 1) et il préfère des comportements inappropriés (valeur négative). Le modèle décrit très bien les comportements du participant.

An hypothetical illustration of social sensitivity. B1 and B2 are referred to as appropriate and inappropriate behavior respectively. $R 1$ and $R 2$ are referred to as obtained reinforcers according to the associated behavior. Sensitivity is 1.13 and is represented by the slope, the bias is -0.09 and is represented by the intercept and the explained variance $\left(r^{2}\right)$ is $78 \%$. The subject is quite sensitive to social reinforcers (>1) and prefers inappropriate behavior (negative value). The model describes subject's behavior well. 
comportements désirables, comparativement à des procédures punitives telles que le retrait d'un privilège ou l'addition d'une tâche pénible, dont le regard s'attarde aux difficultés et aux comportements inadéquats de l'élève. Comme il a été souligné précédemment, la sensibilité sociale propose l'idée selon laquelle les comportements d'une personne ne sont pas fonction de la valeur du taux de renforçateurs absolu qu'il obtient, mais plutôt de leur valeur relative [2]. En d'autres termes, ce n'est pas la quantité totale de renforçateurs qui est pertinente à l'intervention, mais bien la façon dont ils sont distribués par l'intervenant. Cette dernière contribution est d'autant plus accessible aux enseignants puisque ces derniers n'ont pas à augmenter leur taux de renforcement total [1].

Récemment, une recension de la littérature empirique a permis de démontrer la validité de l'application de la loi de l'appariement afin de quantifier la sensibilité sociale [10]. Les résultats de cette recension suggèrent que cette conception peut faciliter l'élaboration de stratégies d'interventions comportementales efficaces et plus individualisées en contexte éducatif. Cependant, les auteurs remarquent que la plupart des études ont été réalisées auprès d'enfants présentant diverses problématiques. De plus, la recension ne met pas l'accent sur les interventions concrètes pouvant découler de la mesure de la sensibilité sociale. Ainsi, l'objectif de la présente étude est d'accroitre les connaissances sur la sensibilité sociale d'élèves tout-venant et d'illustrer différentes interventions avec leurs résultats.

\section{Méthode}

\section{Participants}


Les deux participants sont des enfants d'une école primaire de la région de Montréal. Ils sont âgés respectivement de six et huit ans. Tous deux sont désignés par leur enseignante comme ayant un niveau d'attention à la tâche plus faible que celui de leurs pairs. Toutefois, ils ne présentent aucun diagnostic psychologique. Ils fréquentent une classe ordinaire de première et troisième année primaire.

L'étude est réalisée à l'intérieur d'un programme de formation et de perfectionnement offert aux enseignants qui désirent améliorer leurs habiletés de gestion de classe par un spécialiste de l'analyse du comportement. Les enseignants souhaitant participer à ce programme doivent accepter qu'une observation directe de leurs interventions éducatives soit réalisée par le professionnel. Étant donné que ce dernier agit à titre de consultant, il revient à la direction de l'école et à l'enseignant d'obtenir le consentement des parents des élèves.

\section{Procédure}

L'observateur est un psychologue consultant dont la tâche spécifique est d'améliorer les habiletés de gestion de classe des enseignants. Il a une formation de plus de huit ans en observation directe, et la fidélité interjuges de ses observations a été validée dans d'autres cas. Il est présent en salle de classe et n'intervient pas auprès des élèves ni de l'enseignante. Cette dernière poursuit son enseignement sans interagir avec l'observateur. La technique d'observation par intervalle de 10 secondes est utilisée afin de noter la fréquence d'apparition des comportements et des renforçateurs. Ainsi, l'expérimentateur observe cinq secondes les interventions de l'enseignante et les comportements de l'élève, puis note ses observations pendant les cinq secondes 
subséquentes. Les séances d'observation durent dix minutes. Le nombre de séances varie entre une et deux par jour, compte tenu des situations imprévues pouvant se dérouler en milieu scolaire. Au total, les participants sont observés respectivement 60 et 80 minutes. La procédure s'étale sur une période de deux semaines et de six mois.

\section{Comportements cibles et renforçateurs}

Les comportements sociaux des élèves cibles sont codifiés, observés et notés selon la grille de Forget et Otis [11]. Elle a pour objectif d'être la plus exhaustive possible afin de bien rendre compte des interactions en salle de classe. C'est pourquoi elle prévoit des comportements peu probables, mais susceptibles de survenir. La grille comporte huit catégories de comportements. Les sept premières sont jugées comme étant des comportements inappropriés dans le contexte d'une salle de classe : déplacement inutile, bruit, agression, commentaire, bavardage, comportement moteur, et activité étrangère. La huitième catégorie correspond au comportement d'attention à la tâche et elle est la seule considérée comme étant appropriée. Pour être codifiée, cette classe de comportement doit apparaître si et seulement si aucune autre catégorie de comportements n'est manifestée par l'élève pendant l'intervalle d'observation. Les comportements sociaux des enseignants sont aussi codifiés selon la grille de Forget et Otis [11] qui comprend les comportements verbaux, les regards, les gestes, la proximité, les contacts physiques, l'inscription à l'agenda (carnet de liaison entre l'enseignant, l'élève et ses parents), l'isolement (l'acte de sortir l'enfant de la classe ou de l'isoler dans un endroit spécifique) ou les renforçateurs tangibles. Il faut rappeler qu'une hypothèse sous-jacente au modèle est que l'attention sociale possède une fonction opérante peu importe que l'attention puisse sembler négative ou agréable [7]. Enfin, pour que le comportement de 
l'enseignant soit associé à un comportement de l'enfant, les deux doivent apparaître dans le même intervalle de cinq secondes.

\section{Résultats}

Afin d'évaluer la sensibilité sociale des participants, il faut spécifier les paramètres-utilisés à l'intérieur de la corrélation. D'abord, le comportement approprié « être attentif à la tâche » est abrégé par $B 1$, la classe de comportement cible. Les autres comportements sont symbolisés par B2. Les renforçateurs associés à ces comportements sont identifiés par $R 1$ et $R 2$ respectivement. Ensuite, ces valeurs sont transformées en ratio puis sous forme logarithmique telle que spécifiée par la loi généralisée de l'appariement (voir équation 1) [8]. Une régression est finalement utilisée afin d'estimer la sensibilité $(a)$, le biais $(\log (c))$, et la variance expliquée $\left(r^{2}\right)$ par le modèle. Cette procédure décrite par Reed [12] se réalise facilement à l'aide du logiciel Microsoft Office Excel.

La figure 2 illustre le degré de la sensibilité sociale des participants. Ainsi, le modèle explique respectivement $58 \%$ et $82 \%$ de la variance pour les participants 1 et 2 . Ces résultats sont similaires aux données de la littérature empirique dans le domaine [10]. La sensibilité est cependant différente pour les deux enfants. Ainsi, le participant 1 manifeste une sensibilité de 0,73 , légèrement en dessous de ce qui est considéré comme théoriquement idéal, mais qui correspond à ce qui est habituellement observé auprès de participants humains tant en contexte expérimental [13-14] qu'appliqué [10,14]. Le participant 2, quant à lui, semble peu sensible aux contingences de renforcement social 

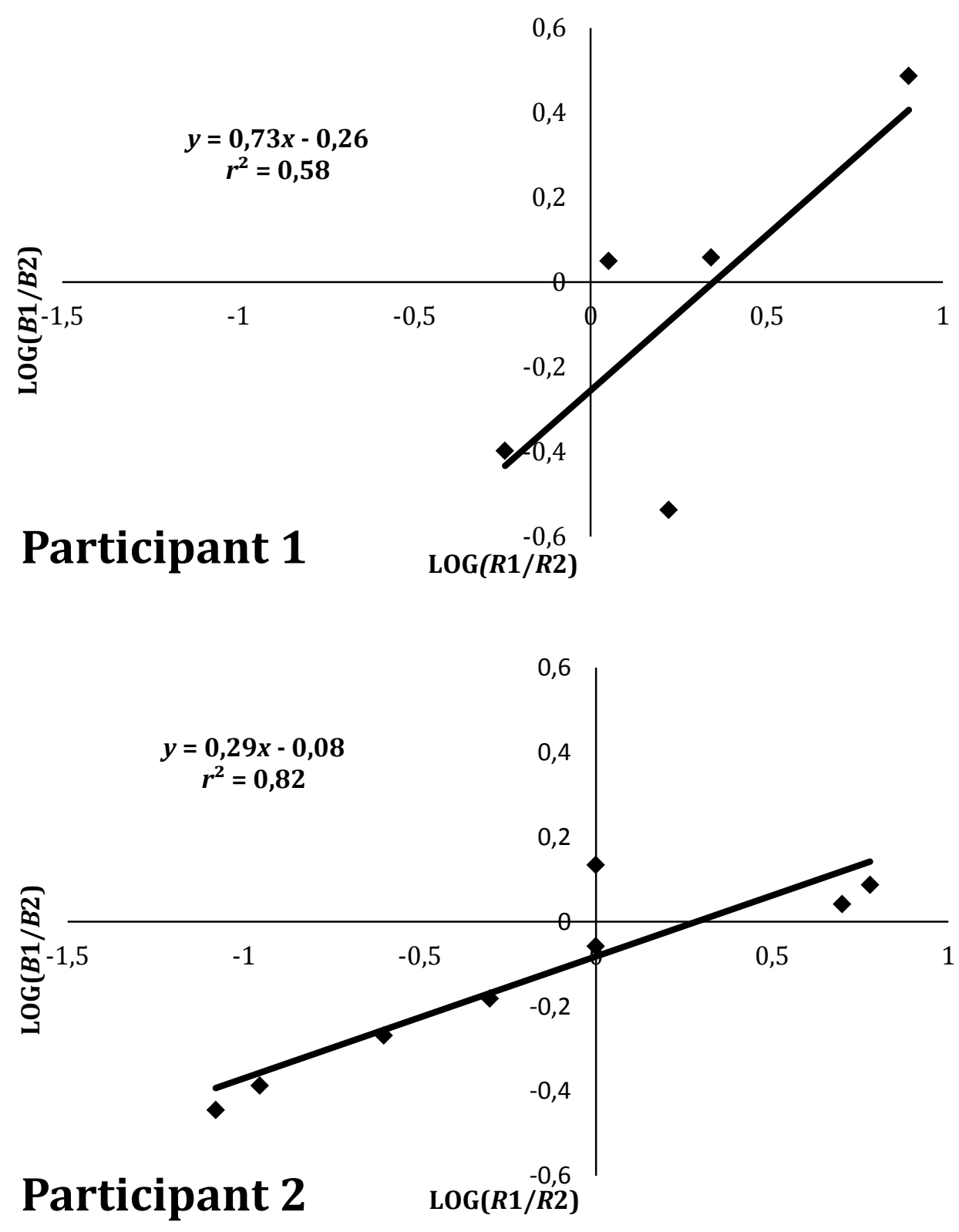

Figure 2. La sensibilité sociale des deux participants. Le cadre supérieur présente les résultats du participant 1 et le cadre inférieur ceux du participant 2. Les valeurs de la sensibilité, du biais et de la variance expliquée sont indiquées à l'intérieur de chaque cadre. Le participant 1 montre une sensibilité inférieure à l'unité et une importante préférence pour les comportements inappropriés. La sensibilité sociale décrit bien les l'allocation des comportements de ce participant. Le participant 2, quant à lui, montre 
presque une insensibilité aux contingences de renforcement social et une préférence presque nulle pour les comportements inappropriés. La sensibilité sociale décrit très bien l'allocation des comportements de ce participant.

Social sensitivity of both subjects. Upper panel depicts results of subject 1 and lower panel depicts results of subject 2. Subject 1 shows a sensitivity lower than one and an important preference to inappropriate behavior. Subject 2 is almost insensitive to the contingencies of social reinforcement and slightly prefers inappropriate behavior. Both subjects' behaviors are well described by social sensitivity. 
avec une sensibilité de 0,29 . Ce résultat est d'autant plus intéressant que la variance expliquée est plus élevée pour ce participant. En d'autres termes, l'ajustement comportemental de l'élève est plutôt faible par rapport aux variations de l'attention sociale de son enseignant, mais le modèle décrit bien sa sensibilité. Enfin, il semble bien que les deux participants présentent une préférence à émettre des comportements inappropriés. Ainsi, le participant 1 manifeste le plus grand biais $(-0,26)$ comparativement au participant $2(-0,08)$. Cela signifie que les participants auront tendance à émettre des comportements inappropriés et que cette tendance demeure constante, peu importe la variation dans le taux d'attention. Le biais du participant 2 est plus négligeable que celle du participant 1 . Ces derniers résultats confirment la perception des enseignants selon laquelle ses élèves ont tendance à ne pas être très attentifs à la tâche.

\section{Discussion}

L'objectif de la présente étude est d'illustrer différentes interventions selon les résultats obtenus par le modèle de la sensibilité sociale. Les variances expliquées élevées montrent que le modèle décrit assez bien les relations entre les comportements d'un individu et l'attention sociale prodiguée par son entourage. Ces résultats viennent, par conséquent, corroborer la littérature empirique. Les participants se conforment aux autres données publiées sur le concept [2, 10, 14]. Enfin, la sensibilité sociale permet de proposer des interventions spécifiques pour chacun des participants.

\section{Suggestion d'intervention pour le participant 1}


Les résultats du participant 1 montrent que la sensibilité sociale décrit bien ses comportements et que celui-ci est sensible à la distribution de l'attention sociale de son enseignant. En revanche, il ne faut pas négliger que le participant a tendance à émettre plus des comportements inappropriés. Ainsi, l'enseignante doit prendre des précautions si elle désire modifier la façon dont elle accorde son attention à l'élève, car une erreur entraînerait probablement une augmentation importante de la fréquence de comportements indésirables. Par conséquent, si l'enseignante cherche à augmenter les comportements adéquats en salle de classe, elle doit être particulièrement alerte et renforcer les comportements adéquats, et ce, de façon contingente à leur manifestation. À titre d'exemple, si l'enfant travaille en silence, il est possible de se diriger derrière et de lui toucher l'épaule discrètement pour l'encourager à poursuivre la tâche demandée. Parallèlement, l'enseignante doit ignorer, dans la mesure du possible, les comportements inadéquats afin de faire diminuer sa fréquence relative. Ainsi, si l'enfant parle sans lever la main, l'enseignante doit ignorer son commentaire plutôt que de le reprendre.

\section{Suggestion d'intervention pour le participant 2}

Les résultats du participant 2 montrent que le modèle décrit très bien les interactions entre les comportements de ce dernier et ceux de son enseignante. En revanche, le participant semble plutôt insensible aux renforçateurs sociaux. Quoique ce résultat semble paradoxal, il faut se rappeler que la variance expliquée permet de vérifier la vraisemblance à laquelle les paramètres peuvent être interprétés et que le paramètre de la sensibilité atteste le degré de sensibilité sociale. Bref, l'interprétation de ces paramètres est relativement indépendante l'une de l'autre. Concernant l'intervention, les résultats du participant 2 signifient que l'enseignante devra augmenter de façon substantielle son taux 
d'attention relatif aux comportements appropriés et devra s'attendre à des changements modestes. Évidemment, selon le modèle, elle devra diminuer de manière importante son attention aux comportements inappropriés afin de diminuer l'apparition de ceux-ci et s'assurer que l'écart (le ratio) entre les taux d'attention donnée aux deux types de comportement soit le plus élevé possible. Il peut sembler contre-intuitif pour un enseignant de ne plus intervenir auprès des comportements inappropriés. Cependant, dans la mesure où l'hypothèse selon laquelle l'attention agit bel et bien comme renforçateur social, le fait de porter attention à un comportement qu'il soit approprié ou non, implique nécessairement un accroissement de sa probabilité de réapparition. À l'inverse, l'ignorance, une procédure d'extinction, correspond à une diminution de cette probabilité. Dans le présent cas, la recommandation est pourtant peu risquée, puisque cet élève manifeste peu de comportements inappropriés. Ainsi, dans une telle situation, le modèle prédit qu'en diminuant le ratio des renforçateurs, l'enfant devrait optimiser ses comportements appropriés.

D’autres hypothèses sont aussi à envisager, mais elles nécessiteraient davantage d'analyses. Ainsi, le participant 2 pourrait être sensible à l'attention prodiguée par les pairs ou la valeur de l'attention de l'enseignant pourrait ne pas être assez intéressante. Les renforçateurs prodigués par les pairs peuvent jouer un rôle important dans le maintien des comportements inappropriés. Dans une telle situation, il devient important de sensibiliser les élèves à l'ignorance intentionnelle des comportements inadéquats de leurs camarades. Aussi, l'hypothèse sous-jacente au modèle selon laquelle l'attention de l'adulte peut avoir une fonction opérante, peu importe qu'elle semble agréable (p.ex. félicitations) ou aversive (p. ex., réprimandes) peut ne pas être toujours valide. Il serait 
alors pertinent d'évaluer autrement la nature de la relation entre l'élève et l'enseignant tel que de mesurer le lien d'attachement entre l'élève et son maitre par l'entremise d'un questionnaire (voir, par exemple, [15]). Si l'enseignant est une personne significative pour l'élève, cela pourrait favoriser la manifestation de comportements adéquats tout en favorisant sa réussite éducative [16]. Dans le cas contraire, il est plus probable que l'attention sociale prodiguée par l'enseignante n'ait pas de valeur renforçante, voire même aversive. Il faudra alors se pencher sur d'autres alternatives. Ainsi, un système d'économie de jetons, l'utilisation de renforçateurs tangibles ou l'utilisation de temps libre contingent à la réussite d'un certain nombre de tâches constituent des stratégies pertinentes lorsque l'intensité ou la fréquence des comportements inappropriés de l'élève sont telles que le fonctionnement général des autres élèves est perturbé. Cela dit, l'usage de renforçateurs matériels ne peut jamais remplacer des déficiences dans l'attribution de l'attention sociale de l'enseignant envers ses élèves. Ces stratégies peuvent accompagner une meilleure distribution de l'attention sociale, mais ne peuvent s'y substituer. Les enseignants et les éducateurs sont parfois réticents à utiliser ces interventions, les percevant comme plus contraignantes ou artificielles. Bien qu'ils doivent être mieux renseignés quant aux résultats probants des thérapies comportementales et cognitives (voir, par exemple, [5-6]), l'utilisation de la sensibilité sociale, telle que présentée ici, peut faciliter leur participation.

\section{Conclusion}

La présente étude montre que la sensibilité sociale peut s'appliquer à des contextes éducatifs et qu'elle permet d'engendrer et développer des stratégies d'intervention individualisées et efficaces. Cependant, ces conclusions doivent être 
interprétées en tenant compte de certaines limites de l'étude. D'abord, la validité des observations n'a pu être établie avec un accord interjuges. L'expérience du psychologue en observation directe peut néanmoins garantir une certaine validité des résultats. Ensuite, il faut préciser que l'observation par intervalle possède certaines limites inhérentes à son utilisation. Il est possible d'observer un renforçateur avant un comportement ou encore de codifier un comportement alors que le renforçateur est attribué pendant l'intervalle de notation. Cette méthode possède cependant des avantages comparativement à l'enregistrement vidéo ou l'observation continue : (1) l'information perdue est systématique plutôt qu'aléatoire (comme c'est le cas pour l'observation continue) et (2) elle est plus pratique, car $(2,1)$ elle ne nécessite pas le consentement libre et éclairé de tous les parents des élèves et $(2,2)$ elle est plus susceptible d'être utilisée par d'autres psychologues (car cette méthode est moins onéreuse en termes de temps). Dans ce contexte, l'observation directe par intervalle s'avère l'outil approprié.

Par ailleurs, le nombre de participants étudiés se limite à deux, il est impossible de généraliser les résultats à une population du primaire, mais considérant que la sensibilité sociale repose sur une conception intra-individuelle [17] et qu'il existe une littérature expérimentale validant le modèle [9-10], le nombre de participants n'est pas ici un facteur essentiel. En revanche, le nombre de données par participant est plutôt restreint. Dans la mesure où la sensibilité sociale appartient à une tradition molaire de l'analyse du comportement (voir [14,18]), cette quantité de données n'est pas particulière [19]. Enfin, la valeur d'un modèle d'intervention se base sur les résultats qu'elle engendre [20]. Il reste donc à favoriser des études plus expérimentales où les taux de renforcement sont systématiquement contrôlés. Néanmoins, il faut rappeler que le contexte d'une salle de 
classe pose des contraintes importantes aux chercheurs qui désirent reproduire des

modèles issus de la recherche fondamentale. Bien qu'il s'agisse là d'un défi à relever, la transaction entre les modèles développés en laboratoire et leurs applications en contexte éducatif saura certainement porter ses fruits.

\section{Déclaration d'intérêt}

Les auteurs déclarent ne pas avoir de conflit d'intérêts en relation avec cet article.

\section{Remerciements}

Le premier auteur est appuyé par le Fonds de recherche du Québec - Société et culture. 


\section{Références}

1. Forget J. Les relations fonctionnelles entre l'attention sociale d'enseignantes et les comportements sociaux d'un élève du primaire. Rev Que Psychol 1987;8(1):27-43.

2. Forget J, Rivard M. Évaluer la sensibilité sociale de l'enfant à l'attention de l'adulte. Perspective de recherche en autisme. In: Magerotte G, Willaye E, editors. Intervention comportementale clinique. Bruxelles: De Boeck; 2010. p. 235-87.

3. Ministère de l'Éducation. Politique de l'adaptation scolaire: une école adaptée à tous ses élèves. Disponible de :

http://www.mels.gouv.qc.ca/fileadmin/site_web/documents/publications/EPEPS/Formati on_jeunes/Adaptation_scolaire/politi00F.pdf

4. Agence européenne pour le développement de l'éducation des personnes présentant des besoins éducatifs particuliers. Intégration scolaire et pratiques pédagogiques. Disponible de : http://www.europeanagency.org/sites/default/files/inclusive-education-and-classroom-practices_iecp-fr.pdf 5. Reder F, Stephan E, Clément C. L'économie de jetons en contexte scolaire: risque d'un effet délétère sur la motivation?. J Ther Comport Cogn 2007;17(4),165-169.

6. Rivard M, Morin D, Clément C. Trouble anxiété de séparation : Cas clinique chez un enfant ayant un trouble grave d'apprentissage. Rev Francoph Clin Comport Cogn $2008 ; 13(4), 18-28$.

7. Sajwaj T, Dillon A. Complexities of an elementary behavior modification procedure. In: Etzel CB, Leblanc JM, Baer DM, editors. New developments in behavioural research Hilldales: Erlbaum; 1977. p. 303-15. 
8. Baum WM. On two types of deviation from the matching law: bias and undermatching. J Exp Anal Behav 1974;22(1):231-42.

9. Davison M, McCarthy D. The matching law: a research review. Hilldales (NJ): Erlbaum; 1988.

10. Caron P-O, Royer F, Forget J. La sensibilité sociale : une recension critique. Acta Comport sous-presse.

11. Forget J, Otis R. La modification de comportements sociaux difficiles chez l'enfant. In: Fontaine O, Cottraux J, Ladouceur R, editors. Cliniques de thérapie comportementale. Liège: Mardaga; 1984. p. 223-44.

12. Reed DD. Using Microsoft Office Excel® 2007 to conduct generalized matching analyses. J Appl Behav Anal 2009;42(4):867-75.

13. Pierce WD, Epling WF. Choice, matching, and human behavior: A review of the literature. Behav Anal 1983;6(1):57-76.

14. Forget J, Donais S, Giroux N. La loi de l'appariement et ses applications en psychologie clinique et en éducation. Rev Psychoeduc Orient 2001;30(2):311-27.

15. Harrison LJ, Clarke L, Ungerer JA. Children's drawings provide a new perspective on teacher-child relationship quality and school adjustment. Early Child Res Quart 2007;22(1),55-71.

16. Desrosiers H, Japel P, Singh PRP, Tétreault K. La relation enseignante-élève positive : ses liens avec les caractéristiques des enfants et la réussite scolaire au primaire. Disponible de : http://www.jesuisjeserai.stat.gouv.qc.ca/pdf/publications/feuillet/ELDEQ_fasc6no2.pdf 
17. Caron P-O. On applying the matching law to between-subject data. Anim Behav 2013;85(4):857-60.

18. Baum WM. Molar and molecular views of choice. Behav Processes 2004;66(3):349-59.

19. Leroux-Boudreault A, Forget J, Caron P-O. Une analyse picoéconomique de l'attention à la tâche d'écoliers et de l'attention de l'enseignant. Acta Comport $2014 ; 22(1): 73-88$.

20. Hanley GP, Iwata BA, McCord BE. Functional analysis of problem behavior: A review. J Appl Behav Anal 2003;36(2):147-85. 


\section{Résumé}

Comment améliorer les comportements d'un élève sans nuire à la gestion globale de la salle de classe ? En fait, la sensibilité sociale constitue un concept privilégié afin d'améliorer les habiletés des enseignants à gérer les comportements difficiles de leurs élèves [1]. La sensibilité sociale est un concept décrivant la relation entre les comportements d'un individu et l'attention sociale prodiguée par son entourage [2]. L'objectif de la présente étude est de présenter ce concept et d'illustrer deux études de cas réalisées selon les résultats obtenus à la suite d'une analyse comportementale de la sensibilité sociale de l'élève. L'observation directe est utilisée afin de noter les comportements sociaux des élèves en salle de classe. Un observateur note la fréquence d'apparition des comportements appropriés et inappropriés en contingence avec les renforçateurs prodigués par leur enseignant. Les résultats montrent que la sensibilité sociale décrit $58 \%$ et $82 \%$ de la variance du comportement des élèves. La présente étude illustre la pertinence de ce concept dans l'élaboration d'interventions comportementales en salle de classe.

Mots-clés : attention à la tâche, salle de classe, loi de l'appariement, sensibilité sociale 


\begin{abstract}
Increasing the appropriate response rate of a target student without producing major drawbacks in the classrooms is a major concern for teachers. Several behavioral interventions have been developed to answer this issue. They often require, however, professional supports and may have the disadvantage to focus only on specific and/or isolated problem behaviors. One way to improve teacher skills in managing student behaviors is through the assessment of their social sensitivity [1]. Social sensitivity quantifies the behavioral adjustment of a subject to contingencies of social reinforcement by means of the generalized matching law [2]. It hypothesizes that social attention has an operant function on student behavior and that their adjustment follow the matching law predictions. The purpose of the current study is to illustrate social sensitivity as an analytical tool for identifying different possible treatments in a school context. Two primary school students and their teachers were directly observed in their usual classroom. The students had no diagnosis nor exhibited any problem behavior. They were chosen because, according to their teachers, they showed poor attention to the task. A behavior analyst recorded the naturally occurring rates of appropriate and inappropriate behaviors of the children and the rate of attention given by their teacher. Behaviors and reinforcers were recorded according to an interval sampling method (five seconds of observing followed by five seconds of recording). Social sensitivity accounted for $58 \%$ and $82 \%$ of the variance. Both subjects demonstrated undermatching and a small preference (bias) toward inappropriate behaviors. Subject 1's sensitivity was .73 (moderate undermatching) whereas subject 2's was .29 (strong undermatching). Biases for both subject were respectively $-\log (.026)$ and $-\log (.08)$, favouring inappropriate
\end{abstract}


behavior and confirming teachers' perception that children had low attention to the task. Results are similar to other experimental and descriptive studies in humans. High explained variance suggests that social sensitivity can be applied in the classroom to direct interventions. Based on the obtained matching relations of each subject, two treatments are discussed. Given the results of subject 1 , the teacher should be careful in choosing the behavior to reinforce, because the subject has a strong tendency to behave inappropriately. The teacher does not have to increase the absolute number of reinforcers, but rather should distribute them according to a ratio favoring appropriate behaviors. Assuring that reinforcers were contingent to appropriate behavior was also emphasized. According to subject 2's results, the teacher should extinguish, as much as possible, reinforcers to inappropriate behavior and increase social attention to appropriate ones. Given a lower sensitivity to social attention, the teacher should expect a modest modification of behavior. Other types of treatment, such as token economy or based on tangible reinforcers, could be considered in this case. Some limits should be considered. First, the number of subject and the sample size were low. Since social sensitivity relies on a within-subject and molar conceptualisation of behavior, they do not appear as essential factors. Second, the treatments proposed herein were not tested. Effects of the intervention could not have been empirically investigated. It would have brought better support to the model and its efficiency in classrooms. The current study suggests that social sensitivity can be used in school context in order to influence and fine tune behavioral interventions. It seems easier to introduce reluctant teachers to behavioral and cognitive therapies. They require less professional support and would provide autonomy to teachers towards classroom management. Finally, more studies are necessary to assess 
the therapeutic contribution of social sensitivity; nevertheless, the current results are promising and indicate the need for further examination of the model.

Keywords: attention to the task, matching law, social sensitivity, classroom 